\title{
Accumulation of oxidative hepatobiliary lesions during experimental opisthorchiasis
}

\author{
Oxana Zaparina \\ ICG SB RAS, Novosibirsk, Russia \\ zp.oksana.93@gmail.com
}

\author{
Anna Kovner \\ ICG SB RAS, Novosibirsk, Russia \\ kovner@bionet.nsc.ru \\ Viacheslav Mordvinov \\ ICG SB RAS, Novosibirsk, Russia \\ mordvin@bionet.nsc.ru
}

\author{
Maria Pakharukova \\ ICG SB RAS, Novosibirsk, Russia \\ NSU, Novosibirsk, Russia \\ pakharukova@bionet.nsc.ru
}

\begin{abstract}
Opisthorchiasis caused by the liver fluke Opisthorchis felineus is widespread on Russian territory, Eastern Europe and Asia. This disease leads to liver dysfunctions: cholecystitis, cholestasis, periductal fibrosis, chronic inflammation, and precancerous changes in the epithelium of the bile ducts (biliary neoplasia). The mechanisms of these processes are not study. In the result of study, we showed time- dependent oxidative lesions accumulation and hepatoprotective effect of various antioxidants by reducing inflammation and biliary neoplasia.
\end{abstract}

Keywords - opisthorchiasis, inflammation, reactive oxygen species, antioxidants, liver fluke

\section{Motivation and Aim}

Opisthorchiasis caused by the liver fluke Opisthorchis felineus in the hepatobiliary system of humans and fish-eating mammals is widespread on Russian territory, Eastern Europe and Asia. This disease leads to liver dysfunctions: cholecystitis, cholestasis, periductal fibrosis, chronic inflammation, and precancerous changes in the epithelium of the bile ducts (biliary neoplasia). The causative agents of these diseases, liver flukes $O$. viverrini and $C$. sinensis, are officially recognized as Group 1 biological carcinogens and are classified as the main risk factors for cholangiocarcinoma [1]. Recently specific oxysterol-like molecules were found to be secreted from the flukes. These molecules might have genotoxic and pro-oxidative properties, increase production of reactive oxygen species, oxidative damage to DNA and precancerous lesions in host cells [2]. The mechanisms of development of this disease have not been studied; however, increased production of reactive oxygen species probably plays an important role in the pathogenesis of opisthorchiasis [3].

To study the possible role of oxidative stress in the pathogenesis of opisthorchiasis we assessed lipid peroxidation and inflammation markers accumulation, DNA damage in infected hamsters from 1 to 18 months postinfection and the effect of antioxidants SKQ1 (10- (6'-Plastoquinonyl) decyltriphenylphosphonium) and resveratrol.

\section{Methods}

Experimental opisthorchiasis in vivo on golden hamsters M.auratus. Using immunohistochemistry and immunoassays, we showed accumulation of inflammatory and oxidative damage markers. Pathological changes in the liver, including inflammation, biliary neoplasia, metaplasia, proliferation of the epithelium of the bile ducts, were assessed using histological analysis. Using real time PCR we measured mRNA expresson of the inflammation and fibrogenesis related genes.

\section{Results}

We showed time-dependent accumulation of inflammatory, lipid peroxidation (MDA, HNE) and oxidative DNA damage (8-OHdG) markers. Moreover, liver histopathology, including inflammation, biliary neoplasia, periductal fibrosis were assessed.

The histopathology of liver i.e. biliary neoplasia, lipid peroxidation markers and serum biochemistry liver damage markers were significantly attenuated by SKQ1 and resveratrol treatment.

\section{Conclusion}

Thus, reactive oxygen species play a pivotal role in the pathogenesis of opisthorchiasis, probably mediating the processes of inflammation and biliary neoplasia. The histopathology of liver i.e. biliary neoplasia and lipid peroxidation markers, were significantly attenuated by SKQ1and resveratrol treatment. Thus, SKQ1 and resveratrol have a hepatoprotective effect.

\section{ACKNOWLEDGMENT}

Supported by the RFBR (19-34-90060-A).

\section{REFERENCES}

[1] Sripa B., Kaewkes S., Sithithaworn P., Mairiang E., Laha T., Smout M., Loukas A. (2007) Liver fluke induces cholangiocarcinoma. PLoS Med. 4(7): 1148-1155.

[2] Gouveia MJ, Pakharukova MY, Laha T, Sripa B, Maksimova GA., Rinaldi G, Brindley PJ., Mordvinov VA., Amaro T, Santos LL, Correia da Costa JM, Vale N. (2017) Infection with Opisthorchis felineus induces intraepithelial neoplasia of the biliary tract in a rodent model. Carcinogenesis. 38(9): 929-937.

[3] [3] Pakharukova MY, Correia da Costa JM, Mordvinov VA. (2019) The liver fluke Opisthorchis felineus as a group III or group I Carcinogen. 4 Open. 2(23): 1-10. 